\title{
Concise Definition of Aviation PNT User Requirements Based on OFD
}

\author{
Yan Wang ${ }^{1}$, Wei Zhao ${ }^{2}$, Su Yu ${ }^{1}$ and YangHe Shen ${ }^{1}$ \\ ${ }^{1} 104$ Youyi Road, Haidian District, Beijing, 100094,China \\ 2211 North Fourth Ring Road,Haidian District,Beijing,100083,China
}

\begin{abstract}
Definition of aviation is the core of PNT system requirements analysis and research, The quality of PNT user requirements definition will directly affect the scientificity and effectiveness of other research work of the system, QFD(Quality Function Deployment) is a systematic technical method and management method. In the process of product development, all activities are driven by users' needs. QFD has been widely used in manufacturing, industrial and commercial military fields, and achieved great results. House of quality(HOQ) is the basic tool for establishing QFD system and essence of OFD method. The typical house of quality framework and analytical solution method can be used not only in the development process of new products, but also in the whole process of analysis in other fields. In this paper, aiming at the concise definition of PNT user requirements, QFD technology is used to build QFD model of PNT user requirements and PNT system technology based on the analysis of existing PNT information requirements and future aviation navigation development trend. In order to facilitate the analysis and simplify the approach of aviation navigation as the user requirements, and GNSS, SBAS, DEM, VOR, NDB, ILS and GBAS as the main system technologies. Finally, the technical quality house model of PNT system of aviation navigation task is given, so as to complete the concise definition of PNT user requirements of aviation users.
\end{abstract}

\section{Introduction}

Positioning, navigation, and timing,called as PNT system, refers to comprehensive utilization of various PNT means, through multi-source fusion, overcoming the shortcomings of a single means, deriving a comprehensive application mode of joint field, unified standards, complementary advantages and enhanced performance, providing accurate, continuous and reliable location, time and speed information. It includes unified space-time benchmark, all kinds of PNT means, application system (equipment), basic technology research and development, joint coordination mechanism, policies, regulations and standards.

The development of PNT system has become the focus of attention of many countries in the world. The United States was the first country to put forward the concept of national PNT system ${ }^{[1]}$, and defined the national PNT system as an important national infrastructure, and has formulated detailed development goals and plans ${ }^{[2]}$. While developing and deploying a new generation of GLONASS satellites, Russia has upgraded its ground-based radio navigation system, carried out research and coordination on the realization of navigation functions by using land-based radar signals, and actively developed PNT system. As early as the end of 1990s, the initial design of Galileo program in EU has included the main basic features of PNT system. However, due to the limitation of budget and management system, the EU has to focus on satellite navigation. Britain put forward the concept of "flexible PNT system"[3], which mainly includes the "guard" plan and the "sentry" plan, both of which are systematically considering the construction and technology development of PNT system.

PNT system requirements analysis and research work is the starting point and basis of PNT system research work, and PNT user requirements concise definition is the core of PNT system requirements analysis research work, the completion quality of PNT user requirements concise definition work will directly affect the scientificity and effectiveness of other research work of the system.

In the early stage of the construction of national PNT system, the United States has carried out a lot of research work on requirements, and has set up a special research team to carry out qualitative and quantitative analysis on the PNT requirements of various users, and iterate repeatedly in the process of architecture, which not only ensures the maximum utilization of resources, but also ensures that the national PNT architecture to a great extent in the direction of optimization and rationality

wangyan198567@126.com 
development. The demand of all kinds of users can be confirmed (including the demand from existing and expected users in 2025).

Most domestic research institutions and industries conduct research on PNT information demand from the perspective of system or industry. For example, the 20th Research Institute of China Electronics Technology Group has conducted analysis and Research on the service capacity, user demand and application scope of China's changhe-2 navigation system, TACAN navigation system, microwave landing navigation system and instrument landing navigation system The target is the military aviation and navigation users in China, and the demand analysis report of related system is formed. According to the current development status of China's civil aviation and the future development forecast to 2025, the Civil Aviation Administration of China investigates and analyzes the navigation requirements of commercial aviation users and general aviation users, and proposes PNT information service capability requirements of Civil Aviation Based on PBN capability in different geographical environments.

At present, foreign countries aim to establish a complete demand model in PNT system requirements research, and at the same time, they have developed some tools, such as spreadsheets and databases, which can maximize the top-level guidance role. However, China's demand research is mainly manual, which is still in the stage of "listing" and fails to establish a complete demand model, which not only has limited efficiency, but also has less supporting role.Therefore, it is of great significance to fully study and learn from the successful experience of foreign countries in PNT system requirements analysis.

\section{Overview of Quality Function Deployment (QFD)}

QFD ( Quality Function Deployment) is a systematic technical method and management method. Its basic idea is: in the process of product development, all activities are driven by the needs, preferences and expectations of users, so that the products can meet the needs of users. The specific method is to transform the requirements of users or markets into quality characteristics and design requirements through a series of matrices Requirements, parts characteristics, process requirements, production requirements and other multi-level deductive analysis methods $^{[4]}$, to provide customers with satisfactory products or services. QFD has been widely used in manufacturing, industrial and commercial military fields, and achieved great results.

The concept of house of quality (HOQ) was put forward by Hauser and Clausing in $1988^{[5]}$. It is also called quality chart or quality table. It is an intuitive binary matrix expansion table. House of quality (HOQ) is the basic tool to establish QFD System and the essence of QFD method. QFD establishes the house of quality through customer demand and the quality characteristics of products or services formulated according to customer needs. It expands and decomposes customer requirements step by step, and transforms them into technical requirements and parts characteristics of products, so as to determine the product control methods in each stage, which is composed of typical house of quality.The framework form and analytical solution method can be used not only in the development process of new products, but also in the whole process of analysis in other fields.

HOQ is a kind of image binary matrix expansion chart. A concise HOQ sequence is shown in the figure below, which is composed of six parts.

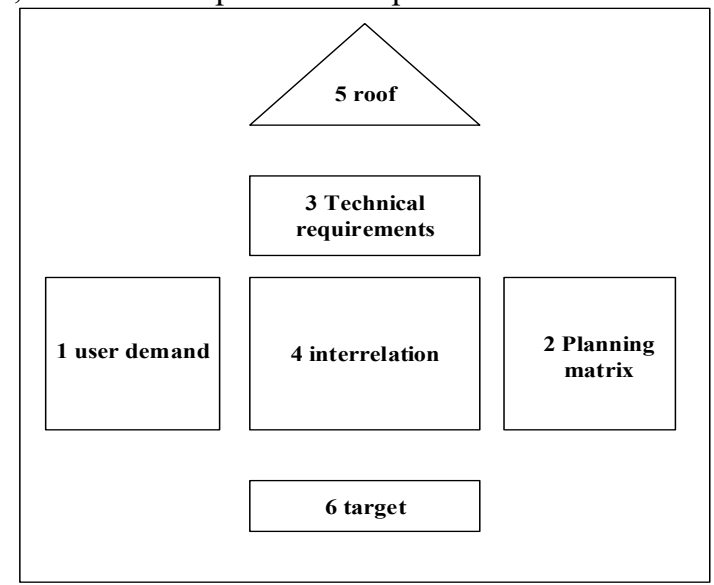

Figure 1. Sequence diagram of house of quality

At present, the most commonly used QFD model is waterfall decomposition model, which decomposes and unfolds user requirements step by step, and transforms them into technical requirements, part features and process characteristics of products, and finally determines the product quality control method, as shown in Figure 2. 


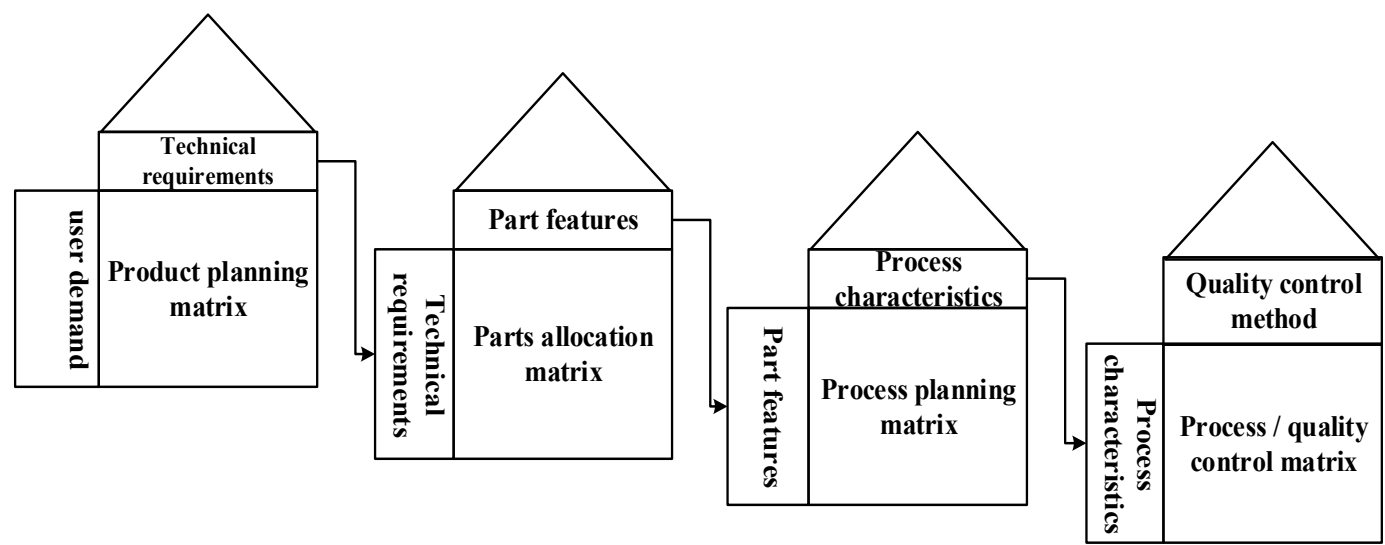

Figure 2. Waterfall decomposition model of QFD house of quality

The typical QFD waterfall decomposition model composed of four house of quality matrices decomposes and configures the user requirements into each process of product development, transforms the user requirements into specific technical requirements and quality control requirements in the product development process, and meets the needs of users by realizing these technical and quality control requirements.

It can be seen that the role of QFD in product development is to transform the user's demand into the final product quality development goal, and the system capability analysis is to transform the strategic demand into the goal of capability development. They have many similarities: 1) they are faced with semi-structured or unstructured decision-making problems; 2) the design characteristics of "products" are determined by the "user" requirements; 3) the requirements of "users" are hierarchical, and with the continuous development of design, the requirements are constantly specified and clarified. QFD starts from the "user" needs and takes the "user" needs into consideration 4) the analysis process provided by QFD method is dynamic, which can reflect the system requirements dynamically and make the development of system capability closer to the actual demand.

\section{QFD Model of system capability requirement analysis}

According to the basic idea of QFD and referring to the waterfall decomposition model of house of quality in the process of product development and design, the analysis model as shown in Figure 3 is established for system capability analysis.

In this model, the system capability analysis starts from the strategic needs, decomposes layer by layer until the capability index that can represent the capability development plan, and finally compares and selects the capability development plan according to the specific capability index and its relationship, which is a top-down analysis and decision-making process. Generally speaking, there are seven levels in the capability analysis model, among which the top-level overall strategic demand comes from the organization's strategic planning the strategic level is the clear development plan proposed by the high-level decision-making department of the organization according to the strategic needs; the task level is composed of the list of key people to be completed to achieve the specific strategic plan; and the capability demand level is based on the relevant "users".The capability level is composed of the capability requirements obtained from the task list; the capability level is composed of the capabilities transformed from the capability requirements; the capability index layer is composed of the capability indicators obtained by the capability decomposition, but it should be noted that the capability index layer may include multiple sub levels, such as two or more levels according to the specific situation; the capability scheme layer is the basis and most important of the whole analysis process, The end goal consists of the capacity development options provided. 


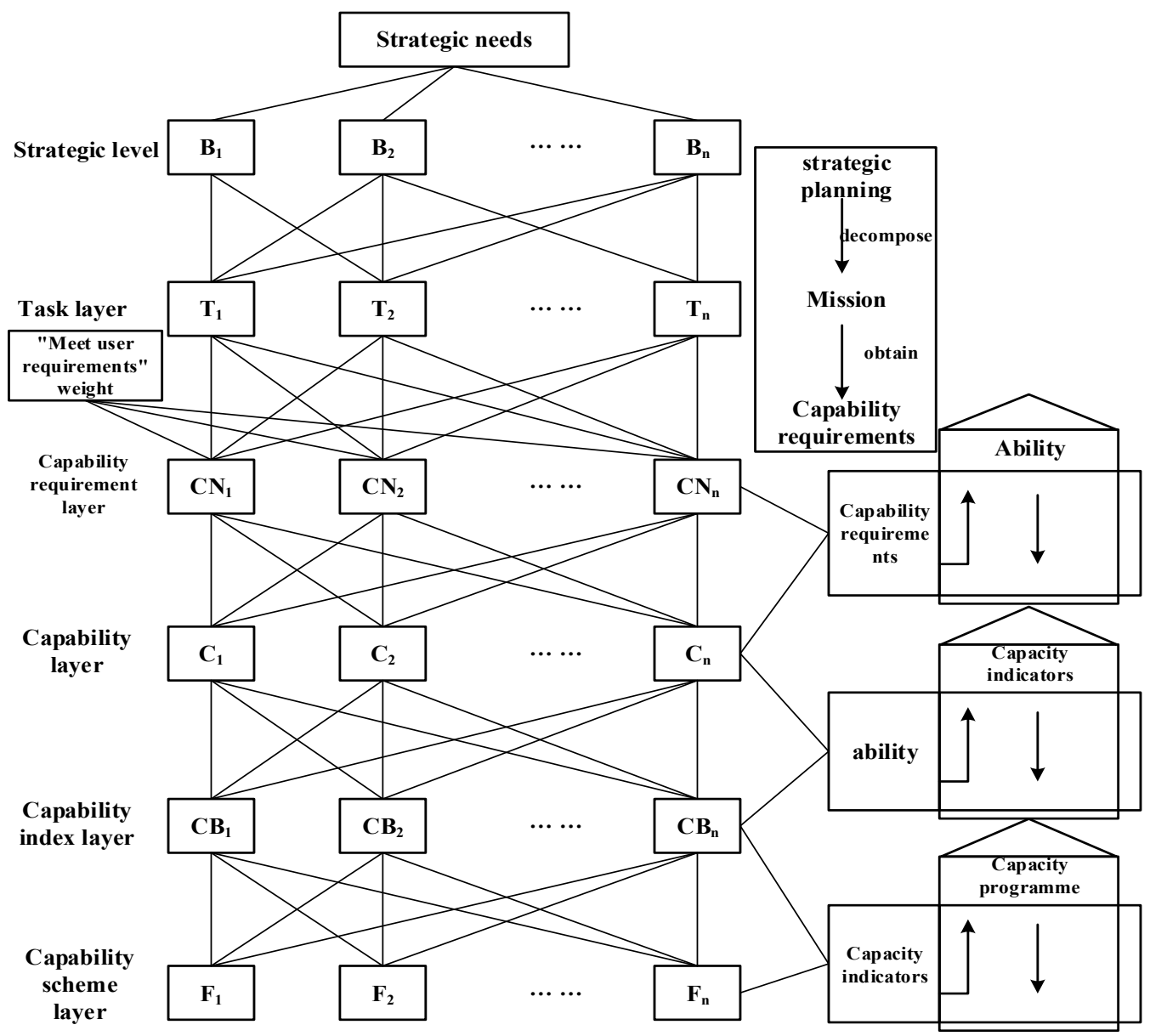

Figure 3. QFD Model of system capability analysis

\section{$4 \quad$ QFD modeling of aviation user PNT requirement refinement}

In this chapter, QFD technology method in Chapter 3 is adopted. Based on the analysis of PNT information requirements of existing aviation navigation and future development trend of aviation navigation, QFD Model of aviation navigation user demand and PNT system technology is established to illustrate the feasibility of this method.

In order to facilitate the analysis and simplify the calculation, this paper takes the requirements of route navigation, non precision approach and precision approach of aviation navigation as the user requirements, and GNSS, SBAS, DME, VOR, NDB, ILS and GBAS as the main system technologies to establish the air navigation mission-PNT system technology house of quality model.

\subsection{Determine the relative importance of "user" requirements}

It is assumed that the mission tasks of aviation navigation include route navigation, non precision approach and precision approach. The implementation steps of AHP are as follows:
(1) Prepare AHP paper and record the navigation mission tasks in the horizontal and vertical columns respectively.

(2) According to the industry benchmark, the mission and task are compared.

(3) Identify experts. Three experts are assumed to be selected here.

(4) Establish expert judgment matrix. If there are three experts, the judgment matrix obtained by the three experts is assumed to be as follows:

$$
\begin{aligned}
& \text { Expert 1: }\left[\begin{array}{lll}
1 & \frac{1}{5} & \frac{1}{9} \\
5 & 1 & \frac{1}{5} \\
9 & 5 & 1
\end{array}\right] \\
& \text { Expert 2: } \\
& {\left[\begin{array}{lll}
1 & \frac{1}{3} & \frac{1}{9} \\
3 & 1 & \frac{1}{5} \\
9 & 5 & 1
\end{array}\right]} \\
& \text { Expert 3: } \\
& {\left[\begin{array}{lll}
1 & \frac{1}{3} & \frac{1}{9} \\
3 & 1 & \frac{1}{5} \\
9 & 7 & 1
\end{array}\right]}
\end{aligned}
$$

(5) The judgment matrix is established:

$$
A=\left[\begin{array}{ccc}
1 & 0.2889 & 0.1111 \\
3.6667 & 1 & 0.1810 \\
9 & 5.6667 & 1
\end{array}\right]
$$


The user importance of the extra large eigenvalue and the corresponding eigenvector are $(0.0646,0.1780,0.7574)$.

According to the calculation formula of the consistency index CI, if CI $=0.0498$, the random consistency ratio $\mathrm{CR}$ of the judgment matrix is $0.0859<$ 0.10 , and the priority ranking of users is reliable.

According to the above calculation, the importance of each user's requirements can be obtained, as shown in Table 1.

Table 1. Calculation of mission importance in different phases of aviation navigation

\begin{tabular}{lccccc}
\hline & $\begin{array}{c}\text { Route } \\
\text { navigation }\end{array}$ & $\begin{array}{c}\text { Non } \\
\text { precision } \\
\text { approach }\end{array}$ & $\begin{array}{c}\text { Precision } \\
\text { approach }\end{array}$ & weight & Consistency test \\
\hline Route navigation & 1 & 0.2889 & 0.1111 & 0.0646 & $\lambda_{\max }=3.0996$ \\
Non precision approach & 3.6667 & 1 & 0.1810 & 0.1780 & $C I=0.0498$ \\
Precision approach & 9 & 5.6667 & 1 & 0.7574 & $C R=0.0859<0.10$ \\
\hline
\end{tabular}

According to the solution of the system capability requirement analysis model, we can get the expansion table of the requirements of each stage of aviation navigation and the requirements of various navigation systems, as shown in Table 2.

Table 2. Expanded table of aviation user navigation tasks and various navigation system requirements

\begin{tabular}{llllllll}
\hline Aviation navigation & GNSS & SBAS & DME & VOR & NDB & ILS & GBAS \\
\hline $\begin{array}{l}\text { Route navigation } \\
\begin{array}{l}\text { Non precision approach } \\
\text { Precision approach }\end{array}\end{array}$ & $\begin{array}{l}\text { Strong } \\
\text { medium }\end{array}$ & $\begin{array}{l}\text { Strong } \\
\text { medium }\end{array}$ & Strong & Strong & Strong & & \\
\hline
\end{tabular}

Note: strong, medium and weak indicate the relationship between task and ability requirement; strong: medium: weak $=9: 3: 1$ or 5:3:1, while blank space indicates that there is no obvious relationship between them.

According to the above aviation navigation user important calculation and user PNT system demand analysis model, and in combination with the characteristics of user capability requirements, a house of quality between the aviation navigation user requirements and the PNT system technology that can be used for aviation navigation is established between the capacity demand list, "user" weight and the importance of capability development as shown in the Figure 4 below.

\subsection{Quality requirements of navigation system between aviation users and PNT}

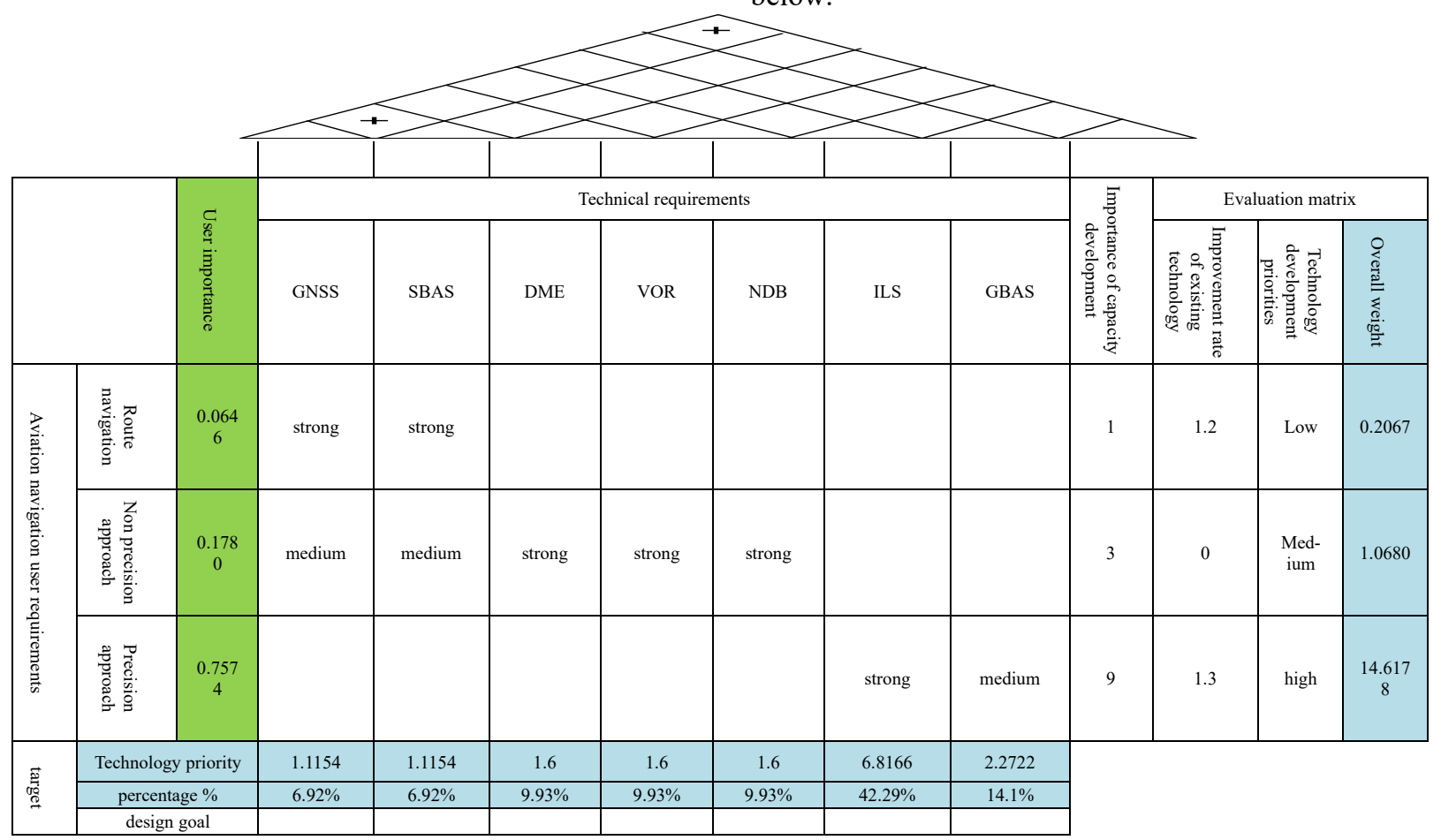

Figure 4. Aviation navigation demand - PNT system technology house of quality relationship diagram 
Note: the priority of capacity development is expressed as high, medium and low. Here high: medium: Low $=$ $5: 3: 1$. The overall weight here $=$ user importance $\times$ (importance of capability development + improvement rate of existing technology + priority of Technology Development)

\subsection{Establishment of house of quality between PNT requirements and existing capabilities of aviation users}

Here, the task phase of aviation navigation user is divided into route navigation, non precision approach, class I precision approach, class II precision approach and class III precision approach. The ability requirements for performing such tasks include horizontal accuracy, vertical accuracy, continuity risk, horizontal alarm threshold, vertical alarm threshold, alarm time and availability.

Suppose that the user importance judgment matrix is:

$$
A=\left[\begin{array}{ccccc}
1 & 1 / 3 & 1 / 5 & 1 / 7 & 1 / 9 \\
3 & 1 & 1 / 3 & 1 / 5 & 1 / 7 \\
5 & 3 & 1 & 1 / 3 & 1 / 5 \\
7 & 5 & 3 & 1 & 1 / 3 \\
9 & 7 & 5 & 3 & 1
\end{array}\right]
$$

Importance of users $\mathrm{W}=(4.4219,4.4486,4.5486$, $4.3552,4.2585,4.4820,3.0000)$. Through determining the objects of investigation, such as the investigation of aviation navigation equipment designers, users and aviation navigation experts, the evaluation criteria of aviation navigation capability index on the Mission relevance of aviation navigation in each stage are determined. Here, strong, medium and weak (strong: medium: weak $=5: 3: 1)$ are used. Through the investigation of domain experts and users, the satisfaction degree of existing capabilities, the improvement rate of existing capabilities, the priority of capacity development, and the economy are determined to evaluate the current capacity service status of aviation navigation in each stage.

Here, the ability satisfaction degree is divided into five levels: "very unsatisfied, unsatisfied, satisfied, over satisfied, very satisfied", namely $\mathrm{M}=[-1,-2,0,1,2]$ The existing capacity improvement rate refers to whether there is room for improvement of service capability, or there is a good way to improve service capacity. It is divided into three levels: "no room for improvement, room for improvement, and room for great improvement", i.e., $\mathrm{R}=[0,1,2]$, and the priority of capacity development is divided into five levels, the higher the value of development level $\mathrm{L}=[1,2,3,4,5]$ The economy can be divided into five grades: the stronger the system economy, the higher the level value, $\mathrm{E}=[1,2,3,4,5]$.

Overall weight $=$ user importance $\times$ (satisfaction of existing capacity + improvement rate of existing capacity + priority of capacity development + Economy).

According to the above calculation results, the relationship diagram of aviation navigation requirements house of quality (HOQ) can be established as shown in Figure 5 .

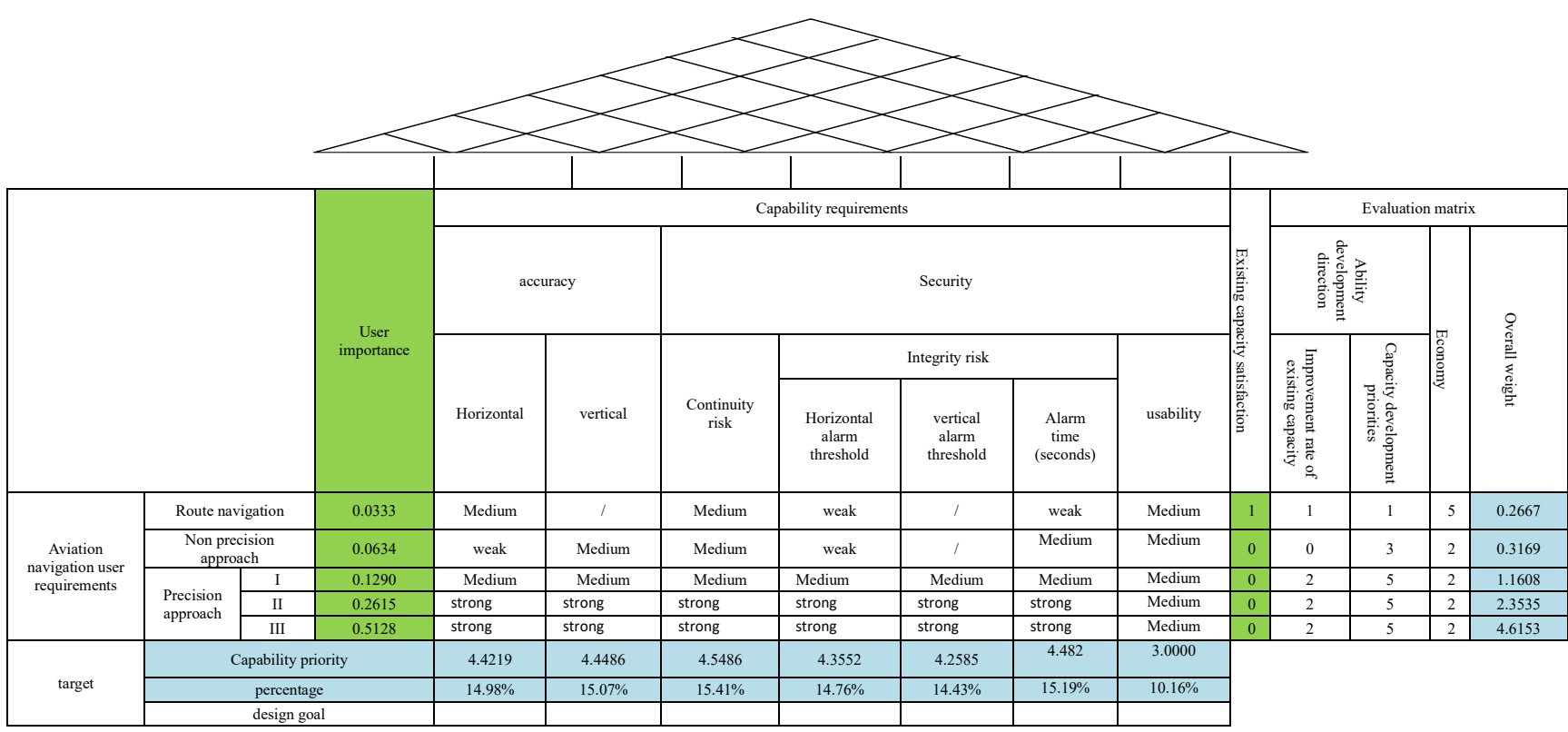

Figure 5. Aviation navigation demand - Capacity demand house of quality relationship

The concise definition of PNT user requirements is the core of PNT system requirements analysis and research,

\section{Conclusion} and is the cornerstone of building a scientific and reasonable PNT system. QFD method and its house of 
quality implementation tools are powerful means to realize the scientific and concise definition of PNT user requirements.

In this paper, the QFD Model of system capability requirement analysis is established. According to the definition of PNT requirements of aviation users, the house of quality between aviation navigation users' requirements and PNT system and the house of quality between aviation users' PNT requirements and existing capabilities are established respectively. The PNT requirements of aviation users are quantified scientifically, which provides a reference for subsequent PNT construction of aviation users, It also provides a useful reference for other users to refine the definition of PNT requirements.

\section{References}

1. ZhiHe Qiu. A preliminary view on the new GPS policy of the United States in 2004[J], Global positioning system,2005,31(3):36-42.

2. DoD\&DOT. National Positioning Navigation and Timing Architecture implement Plan[M]. 2010

3. GREGORY J, SWASZEK P,ALBERDING J et al. The Feasibility of R-Mode to Meet Resilient PNT Requirements for E-navigation [C] Proceedings of the 27th International Technical Meeting of the Satellite Division of the Institute of Navigationg. Tampa Florida: ION 2015 3076-3100.

4. Sullivan L P. Quality Function Deployment. Quality Progress, 1986,19( 6) : 39 50.

5. Hauser J R, et al. The House of Quality. Harvard Business Review,1988,May-June: 63 73. 